\title{
Investigation of Ultra-wideband Bowtie Antennas for Phased Array Feed Application
}

\author{
Jin Fan, Jian Yang, Senior Member, IEEE, Yihua Yan, Wan-Chun Liao, Wai Yan Yong and Dezhi Zhan
}

\begin{abstract}
In this paper, two new ultra-wideband (UWB) dualpolarized Bowtie antennas are investigated as the elements for a phased array feed for reflectors. In addition to its UWB impedance matching characteristic, the Bowtie antennas have stable large beam-width and a low cross-polar level over a wide frequency band with a compact size, which is an essence for phased array applications. The simulated and measured results state a low ohmic loss, good impedance matching $\left(S_{11}\right.$ below -15 dB) and good radiation performance, with a simple structure for easy manufacturing. The proposed antennas can be good candidates for Phased Array Feed (PAF) in FAST and the SKA (Square Kilometer Array) pathfinder PHAROS2 projects, and massive MIMO antennas in wireless communication systems.
\end{abstract}

Index Terms-UWB, bowtie, phased array, phased array feed.

\section{INTRODUCTION}

Recently, phased arrays have been receiving increasing attention in many ultra-wideband (UWB) applications, such as multi-function defense and security radars, wireless communication systems and radio astronomy [1][2]. Phased array feed (PAF) is a kind of novel feed for radio telescope consisting of a large number of small antenna elements, as shown in Fig.1, through the beam synthesis network, the signals received by the array elements are weighted and synthesized with controllable amplitude and phase, forming a number of instantaneous beams [3][4].

The characteristics of the array element can significantly define the achievable performance of the designed whole array antenna or reflector antenna feed array. The selection of array elements plays a crucial role in the phased array design. Conventional radiating elements based on printedon-circuit-board (PCB) can achieve only moderate bandwidths [5-7]. In order to meet the requirement of UWB systems, various UWB array technologies have been developed [8-11]. One of the most popular UWB arrays is the tapered-slot array, also referred to as Vivaldi array [8][9]. Despite excellent wideband performance, the profile height of Vivaldi arrays are typically longer than one wavelength at the low-end frequency. In addition, dual-polarized Vivaldi arrays are not amenable to modular construction because of contiguous electrical connection between their adjacent elements, which raise the array assembly cost and make it difficult to repair if some elements are not functioning well.

This work was supported by Joint Research Fund in Astronomy (U1931129 and U1831117) under cooperative agreement between the National Natural Science Foundation of China (NSFC) and Chinese Academy of Sciences (CAS), NFSC of Grant 11403054 and NSFC-STINT Grant 11611130023.

Jin Fan, Yihua Yan and Dezhi Zhan are with the Department of Key laboratory of Radio Astronomy, the National Astronomy Observatory of China, Beijing 100012, China (e-mail: jfan@bao.ac.cn).
Modular Vivaldi array variations, such as the body of revolution (BOR) Vivaldi array [10], were introduced and could overcome some shortcomings of the original Vivaldi array, but the manufacture of the BOR Vivaldi array antennas requires special machining and it is difficult to implement the array at high frequencies. Tightly coupled dipole arrays (TCDAs) have low profile, wide bandwidth and good scan performance as well as low cross polarization [11]. However, $180^{\circ}$ hybrids with similarly wide bandwidths are required, which introduce additional ohmic and dielectric loss and therefore increase noise, an unfavorable effect in high sensitivity systems such as radio telescopes. The applications of these existing technologies are more or less limited due to either their large physical size, or high polarization level, or manufacture difficulties or increased ohmic loss.

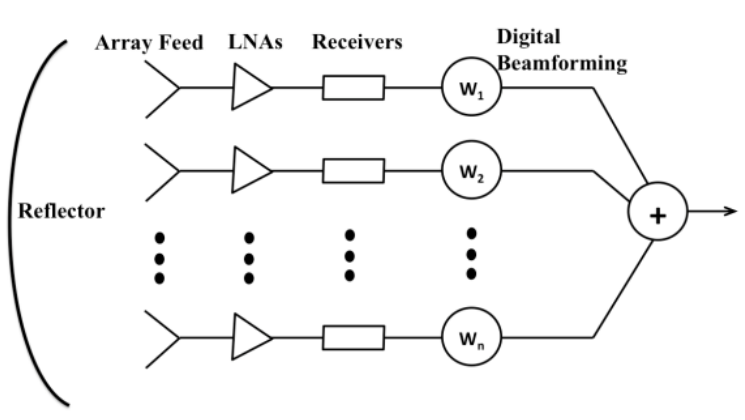

Fig.1. Schematic diagram of phased array feed (PAF)

In the present work, we aim at designing UWB antenna elements as candidates for wideband phased arrays over 4 $8 \mathrm{GHz}$ that can be applied in both wireless communication systems and radio telescopes. First, a compact dualpolarized Bowtie antenna tightly fed by two 50-Ohm coaxes with a set of cross feeding on PCB is presented with a reflection coefficient below $-15 \mathrm{~dB}$ but aberration of radiation patterns at high frequencies in the band. In order to improve the radiation properties, a new antenna element, dual-polarized capped tight Bowtie with a cross feeding, is introduced, which has achieved a stable radiation pattern performance and reduced cross-polarization level over the octave bandwidth. As the mechanism is described, the wideband bowtie prototypes are designed and fabricated to provide experimental verification on the predicted results. The focus of the paper is to present the performance of the

Jian Yang and Wan-Chun Liao are with Department of Electrical Engineering (Antenna Group), Chalmers University of Technology, Gothenburg, Sweden (e-mail: liao@chalmers.se, jian.yang@chalmers.se).

Wai Yan Yong is with Department of Electrical Engineering (Radio System Group), University of Twente, Enschede, Netherlands. (e-mail: w.y.yongwaiyan@utwente.nl). 
array elements, where the performance of whole array system or PAF system will not be included here. The novelty of this work is the investigation of the performance of two new types of Bowtie antennas: the tightly-fed dual-polarized Bowtie and the tightly-fed dual-polarized capped Bowtie, both are directly fed by coaxes without $180^{\circ}$ hybrids balun feedings.

\section{GEOMETRY AND DESIGN PROCEDURE}

\section{A. Structure of Dual-polarized tightly-fed Bowtie}

A sketch of the antenna unit cell is shown in Fig. 2, which is composed of a pair of Bowtie in a cross position, two coaxial lines, two shorted cylinders and a PCB with two orthogonal feeding lines and a common ground plane.

The feeding of the antenna is realized by using two coaxial cables and crossover copper strips on a PCB. The outer conductor of each 50-Ohm coaxial line for each polarization is connected to one Bowtie arm and ground plane. The center core of coaxial cable (with a diameter of $0.51 \mathrm{~mm}$ ) is connected to the opposite metallic Bowtie arm through one of the cross-over microstrip lines (the width of the strips for two orthogonal polarization are $B_{1}$ and $B_{2}$ respectively) which are built on a two-layer Rogers 5880 PCB laminates with a relative dielectric constant of 2.2 and a thickness of $0.254 \mathrm{~mm}$ (size of $D \times D$ ). The other ends of the coaxial cables are connected to SMA connectors which are located underneath the ground plane, opening a possibility to connect the antenna to single-end low-noise amplifiers (LNAs) directly. The ground plane is employed as a reflector for the antenna to produce a unidirectional radiation pattern and the distance between the Bowtie arms and the ground plane is selected as about $\lambda / 4$ (where $\lambda$ is the wavelength at the low end of the frequency band, e.g., $4 \mathrm{GHz}$ ). Detailed design parameters of the antenna are defined as in Fig. 2.

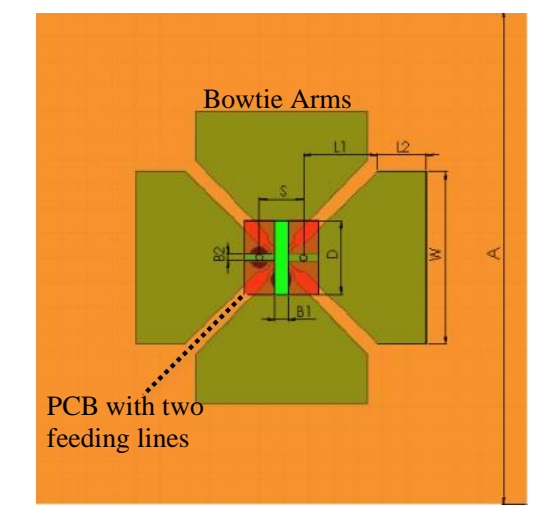

(a)

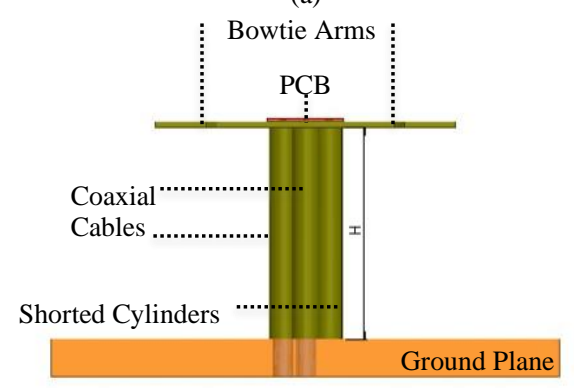

(b)

Fig.2. Geometry of the tightly fed Bowtie antenna (a) Top view (b) Side view

\section{B. Parameter Analysis}

The various parameters have been studied with the assistance of full-wave electromagnetic software Ansys HFSS. A tightly fed Bowtie with the perfectly matched layer (PML) boundary is modeled and simulation analysis is presented from Fig 3 to Fig 6 for the impact of the key parameters on the performance.

Figs. 3 shows the effect of dipole length $\left(L_{1}\right.$ and $\left.L_{2}\right)$ on the reflection coefficient. At first, $L_{2}$ is fixed at $4 \mathrm{~mm}$ and the value of $L_{l}$ is changed from $5.5 \mathrm{~mm}$ to $7 \mathrm{~mm}$ with interval of $0.5 \mathrm{~mm}$. As $L_{1}$ gets increased, it's very intuitive that the operation band with $\mathrm{S}_{11}$ below $-10 \mathrm{~dB}$ is moved to lower frequency because of a larger electrical length of the Bowtie arm. When $L_{l}=5.5 \mathrm{~mm}$, there are two resonances in the operation band. As $L_{l}$ increases to $6.0 \mathrm{~mm}$, another resonance appears around $6 \mathrm{GHz}$. When $L_{l}=6.5 \mathrm{~mm}$, the middle and upper resonances get closer but three resonances still can be recognized. When $L_{l}=7 \mathrm{~mm}$, the middle and upper resonances are merged and only two resonances can be seen. Then the optimal value of $\mathrm{L}_{1}$ is between $6.0 \mathrm{~mm}$ and $6.5 \mathrm{~mm}$ in order to have three resonances for wideband. Secondly, $L_{2}$ are changed between $3 \mathrm{~mm}$ and $6 \mathrm{~mm}$ with $L_{1}$ fixed at $6 \mathrm{~mm}$. When $L_{2}$ increases from $3 \mathrm{~mm}$ to $6 \mathrm{~mm}$, the shift of the low resonance around $4 \mathrm{GHz}$ is more obvious than that of the upper resonance around $8 \mathrm{GHz}$. It can be also seen that with $L_{1}$ fixed at $6 \mathrm{~mm}$, no matter how $L_{2}$ changes, there exist always three resonances. So $L_{2}$ can be also used for tuning of center frequency and in-band return loss.
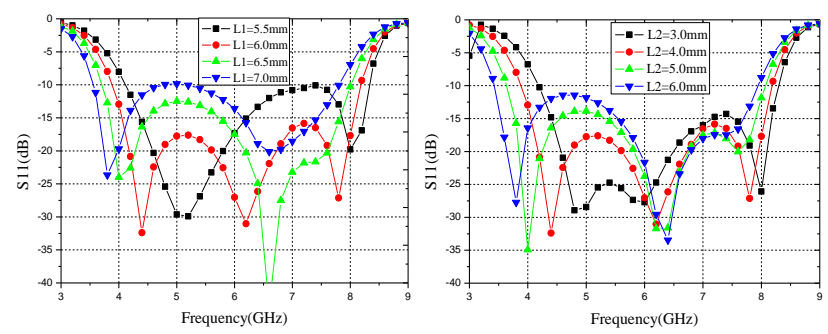

Fig.3. Reflection coefficient with different $L_{1}$ and $L_{2}$
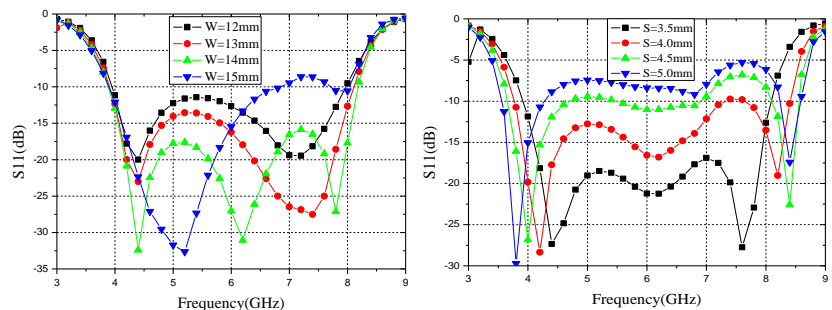

Fig.4. Reflection coefficient with different $W$ and $S$

Fig. 4 illustrates the effect of Bowtie's width $(W)$ and the separation $S$ between the two Bowtie arms varying on the reflection coefficient. We can see that $W$ has little impact on the bandwidth but the in-band impedance matching property and the position of the middle resonance. So it can be used for fine tunings of in-band properties. It is clearly seen that the locations of lower and upper resonant peaks are very sensitive to the variation of $S$, the distance between the center of the feeding coax and the center of the opposite cylinder. When $S=5 \mathrm{~mm}$, the lower resonance can be observed at 3.8 $\mathrm{GHz}$ and higher resonance is at $8.4 \mathrm{GHz}$. When $S$ decreases from $5 \mathrm{~mm}$ to $3.5 \mathrm{~mm}$, the lower and higher resonances are shifted to $4.4 \mathrm{GHz}$ and $7.6 \mathrm{GHz}$ respectively with another resonance occurring around $6 \mathrm{GHz}$. When $S$ is $3.5 \mathrm{~mm}$, the in-band reflection coefficient is below $-15 \mathrm{~dB}$. It can be 
concluded that the bandwidth and impedance properties can be controlled by parameter $S$, but the center frequency does not change much with $S$. So when the center frequency is determined by $L_{1}$ and $L_{2}, S$ can be used to adjust the bandwidth and in-band return loss.

Besides these key parameters mentioned above, the width of feeding strip line ( $B_{1}$ and $\left.B_{2}\right)$ could be adjusted for in-band impedance matching, which will not be elaborated for brief in this part. The final dimensions of the designed tightly fed Bowtie are listed in Table I.

TABLE I

DIMENSIONS OF THE PROPOSED BOWTIE ANTENNA

\begin{tabular}{cc|cc} 
Parameter & Value $(\mathrm{mm})$ & Parameter & Value $(\mathrm{mm})$ \\
\hline$W$ & 14.0 & $B_{I}$ & 1.0 \\
$L_{1}$ & 4.0 & $B_{2}$ & 0.6 \\
$L_{2}$ & 6.0 & $A$ & 25.0 \\
$S$ & 3.6 & $H$ & 17.0 \\
$D$ & 4.5 & & \\
\hline
\end{tabular}

\section{Properties of tightly-fed Bowtie}

The simulated $S$-parameter of the tightly fed Bowtie is shown in Fig. 6(a) in solid lines. The reflection coefficient $S_{11}$ and $S_{22}$ are lower than $-15 \mathrm{~dB}$ and mutual coupling between orthogonal ports is better than $-24 \mathrm{~dB}$ for the band 4.0 GHz-8.0 GHz. Fig. 7(a) give the simulated results of copolar radiation patterns in $E$ - and $H$ - plane as well as crosspolar level in $D$-plane at 4, 6 and $8 \mathrm{GHz}$ respectively.

As the solid lines shown, the radiation patterns of the antenna are relatively symmetrical in the operating bandwidth. However, aberration of radiation pattern emerges at $8 \mathrm{GHz}$ with a serious gain drop at the bore sight, which may result from high-order modes at the high frequency. Besides this, the distance between the dipole and ground plane may be another cause of the aberration in radiation pattern at high frequency band. Based on the image theory, the image electrical current has identical amplitude and opposite phase to the electrical current on the Bowtie arms so as to satisfy the boundary condition that the tangential electric field on ground plane is zero. Current model of the bowtie dipole antenna on the ground plane is as shown in Fig. 5 as well as the equivalent model.

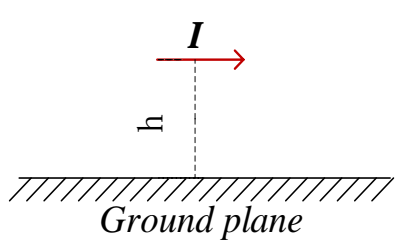

(a)

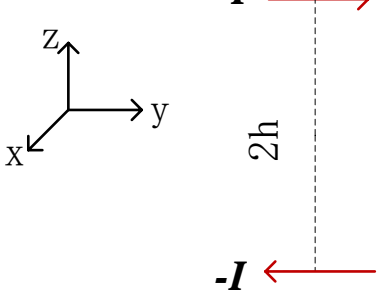

(b)
Fig.5. Current model and equivalent model of the tightly-fed Bowtie (a) Current model of the bowtie dipole on the ground plane (b) Equivalent model

The radiation characteristics of tightly-fed bowtie antenna can be analyzed by a two-element antenna array using Equation (1), where AF is the array factor, $\theta$ is the angle between the radiation direction and the $\mathrm{Z}$-axis and $\pi$ describes the opposite phase between bowtie dipole currents and image currents.

$$
A F=1+e^{i} e^{i \underline{2} 2 h \cos }
$$

At $4 \mathrm{GHz}$, the distance $2 h$ between the electric current on the Bowtie arms and their image current equals to 0.453 $\lambda_{4 \mathrm{GHz}}$, which almost offsets the opposite phase, resulting in a constructive superposition of the far field strength at bore sight $(=0)$ by $10 \log (1+\exp (\mathrm{j}(+0.906)=2.96 \mathrm{~dB}$. However, at $8 \mathrm{GHz}$, the distance $2 h$ between the current and the image current equals to $0.906 \lambda_{8 \mathrm{GHz}}$. With the out of phase for the imaging current, far field strength of the current and the image current nearly counteracts each other by $10 \log (1+\exp (\mathrm{j}(+1.812))=-2.35 \mathrm{~dB}$, resulting in the aberration of the radiation pattern near the bore sight at 8 $\mathrm{GHz}$. On one hand, the gain of this Bowtie antenna is quite stable due to the ground plane over the octave bandwidth. On the other hand, the bandwidth is limited by the ground plane since at $8.8 \mathrm{GHz}, 2 h$ equals to $\lambda_{8.8 \mathrm{GHz}}$ and a null appears for the bore sight radiation.

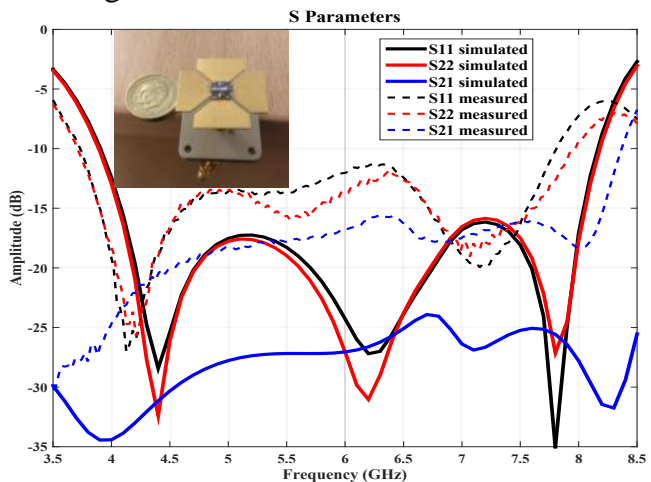

(a)

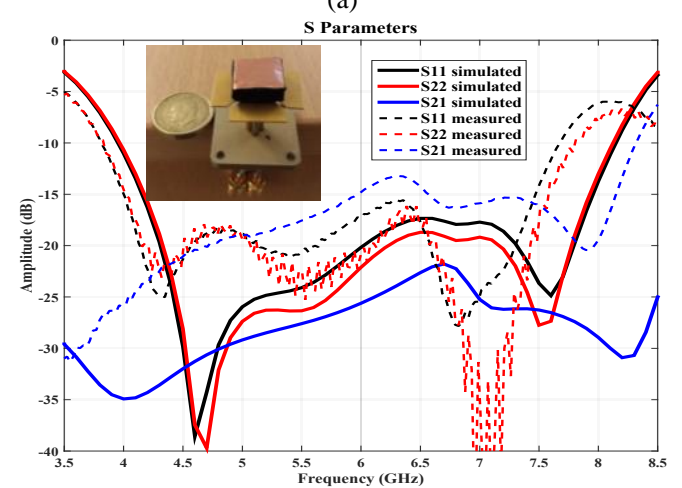

(b)

Fig.6. S-parameters (a) Tightly-fed Bowtie (b) Capped Bowtie

\section{Dual-polarized capped Bowtie}

Tightly fed Bowtie antenna has a critical dilemma which limits the bandwidth: A short height $H$ makes a good radiation patterns at high frequency since the gain drop can be avoided but the reflection coefficient degrades; a large height $H$ provides a low reflection coefficient but a gain drop at high frequencies.

In order to improve the radiation properties without sacrificing impedance bandwidth, a capacitive loading of a parasitic metal patch (a cap) with a dimension of $12 \mathrm{~mm} \times 12$ $\mathrm{mm}$ above the tightly fed Bowtie arms is introduced, embedded in Fig. 6 (b), which shows the simulated $S$ parameters of the capped Bowtie in solid lines. Reflection coefficient $\left(S_{11}\right.$ and $\left.S_{22}\right)$ below $-15 \mathrm{~dB}$ and the mutual coupling between the orthogonal polarizations $\left(S_{21}\right)$ below $22 \mathrm{~dB}$ have been achieved over 4.0-8.0 GHz.

Fig. 7 (b) show the simulated co-polar radiation patterns in $E$ - and $H$-planes as well as cross-polar level in $D$-plane at 
4, 6 and $8 \mathrm{GHz}$ when the capacitive loading placed $6 \mathrm{~mm}$ above the Bowties. As seen in Fig. 8 (b), the capacitive loading has effectively eliminated the aberration in the radiation pattern at $8 \mathrm{GHz}$.
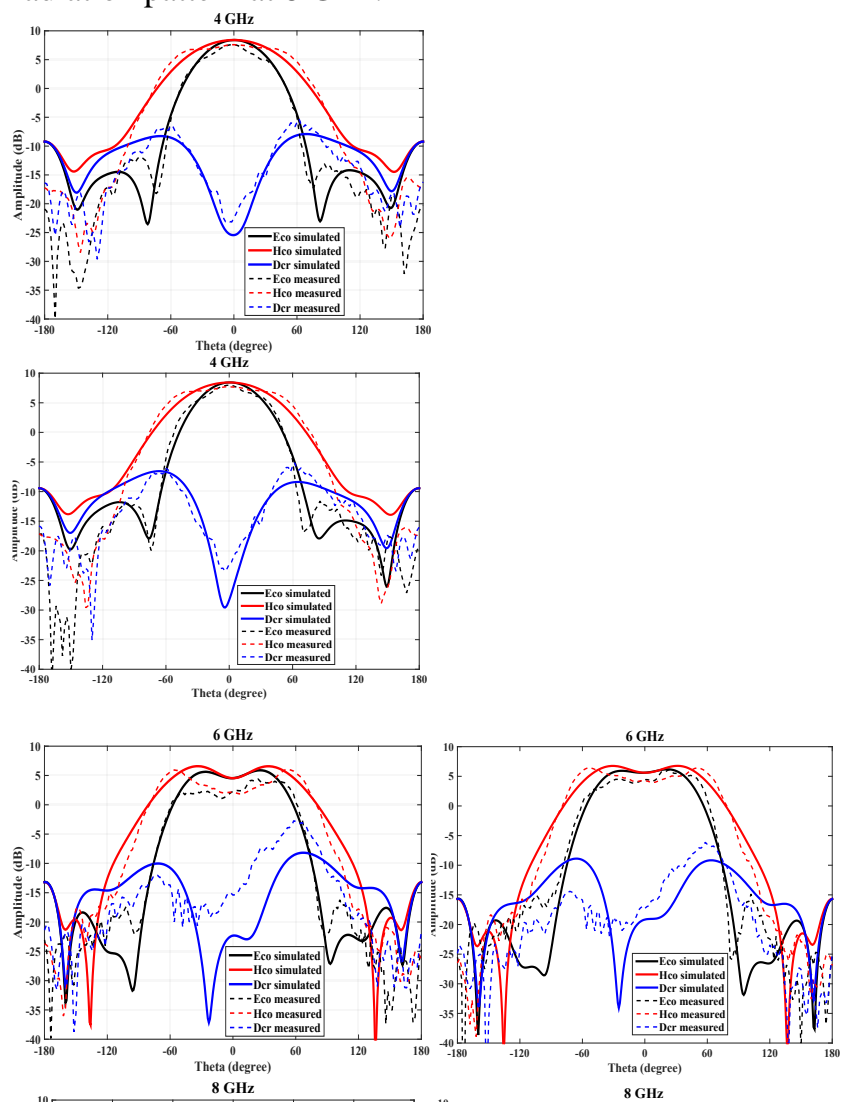

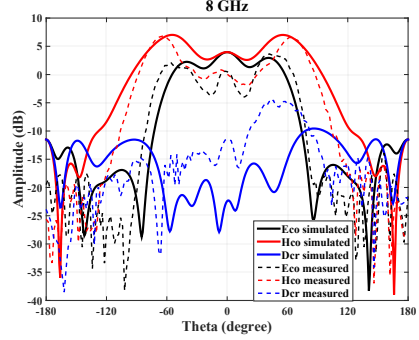

(a)

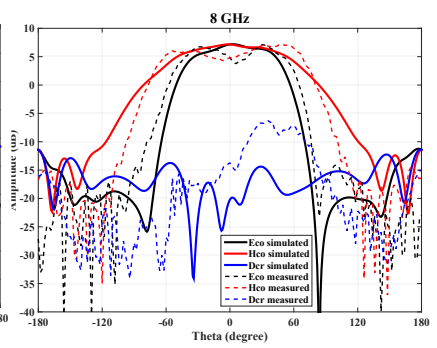

(b)
Fig.7. Measured Radiation Patterns of the Bowtie antenna (a) Tightly-fed Bowtie (b) Capped Bowtie

The radiation characteristics of capped bowtie antenna can be analyzed by a four-element antenna array based on the image theory as shown in Fig. 8. Similar to analysis of the tightly-fed bowtie, the array factor can be expressed as Equation (2), in which the distance of the bowtie and the cap $l$ is chosen around $0.25 \lambda_{8 \mathrm{GHz}}$ and is the current coupling factor smaller than 1 .

$$
A F=2 \sin \left(\frac{2}{h} h \cos \right)+2 \sin \left(\frac{2}{h}(h+l) \cos \right)
$$

The mechanism for wideband performance by this method is that a Bowtie antenna, a Yagi antenna and a stacked patch antenna are combined in an optimal way. Stacked patches can increase the bandwidth of patch antennas and thus applied to a bowtie antenna, which in turn ignites the working principle of Yagi antenna. At low frequencies (around $4 \mathrm{GHz}$ ), $h$ is about a quarter of the wavelength, and the radiation is from the bowtie arms, where the cap (much smaller than a half wavelength) works as a capacitor for impedance matching. At high frequencies (around $8 \mathrm{GHz}$ ), $h$ is very close to half of the wavelength, the first item of Equation (2) on the right side is almost 0 and the second item is close to 2 near the bore sight $(=0)$, where the cap works as a radiating patch while the bowtie arms work as a feeding for the cap. Therefore, at low as well as high frequencies, the cap Bowtie element radiates with almost constant beam. At the middle range of bandwidth, the geometry makes a Yagi antenna with a reflector by the ground plane, a driven element by the Bowtie arms and a director by the cap whose function is to keep the beam constant.

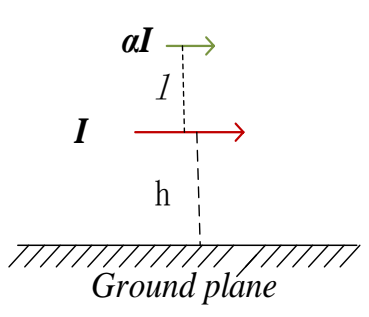

(a)

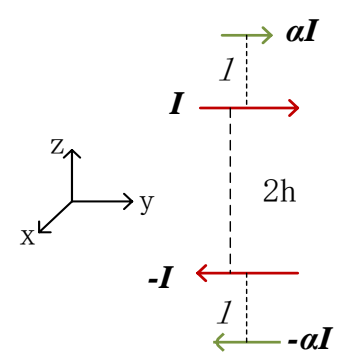

(b)
Fig.8. Current model and equivalent model of the capped Bowtie (a) Current model of the capped bowtie on the ground plane (b) Equivalent model

\section{PROTOTYPE AND MEASUREMENT}

Prototypes of the proposed antennas are fabricated to validate the design. Fig. 6 shows the measured S parameters of the tightly fed Bowtie and capped Bowtie antennas in dotted lines. Owing to the accuracy of the fabrication tolerance and the soldering, difference between the simulated port isolation and the measured one occurs with the frequency range shifting lower and in-band reflection coefficient and mutual coupling higher. Nevertheless, $S_{11}$ and $S_{22}$ below $-15 \mathrm{~dB}$ over an octave bandwidth can still be realized for the capped bowtie.

The simulated and measured radiation patterns for the tightly fed bowtie and capped bowtie antenna are depicted in Fig. 8 in dotted lines. The main lobe will be split when the frequency is higher than $6 \mathrm{GHz}$ if the bowtie antenna is without a parasitic capacitive loading, while nearly stable directional radiation patterns are maintained up to $8 \mathrm{GHz}$ for the capped bowtie. Due to the measurement error from mounting poster, the measured maximum cross-polar levels are higher than the simulated ones, especially at high frequencies.

Tables II shows comparisons of performance between the tightly fed Bowtie and the capped Bowtie. From the patterns and tables the followings can be concluded: 1) Both Bowties have wider H-plane beams than the E-plane ones. 2) The capped Bowtie has more symmetrical patterns much more constant beam-width over the frequency band, especially at high frequencies from 7 to $8 \mathrm{GHz}$ than the Bowtie does. 3) The capped Bowtie has higher gain and lower cross-polar level than the Bowtie does. 4) The tightly fed Bowtie has a broader beam-width than the capped Bowtie does.

Compared to the proposed antennas in [12-16], the bowtie antennas in this paper have a good comprehensive performance of the bandwidth, return loss, and gain level with a compact size as shown in Table III.

Table II

\begin{tabular}{c|c|c|c|c}
\hline \hline \multirow{2}{*}{$\begin{array}{c}\text { Frequency } \\
(\mathrm{GHz})\end{array}$} & \multicolumn{2}{|c}{ 3-dB beam-width $\left(^{\circ}\right)$} & \multicolumn{2}{c}{ Gain and Cross-polar level $(\mathrm{dB})$} \\
\cline { 2 - 5 } & Tightly fed & Capped & Tightly fed & Capped \\
& Bowtie & Bowtie & Bowtie & Bowtie \\
\hline
\end{tabular}


$<$

\begin{tabular}{|c|c|c|c|c|c|c|c|c}
\hline & $\begin{array}{c}\text { E- } \\
\text { Plane }\end{array}$ & $\begin{array}{c}\text { H- } \\
\text { Plane }\end{array}$ & $\begin{array}{c}\text { E- } \\
\text { plane }\end{array}$ & $\begin{array}{c}\text { H- } \\
\text { Plane }\end{array}$ & Gain & $\begin{array}{c}\text { Cro- } \\
\text { polar }\end{array}$ & Gain & $\begin{array}{c}\text { Cro- } \\
\text { polar }\end{array}$ \\
\hline 4 & 61.7 & 90.5 & 61.8 & 90 & 7.6 & -12.2 & 7.7 & -13.8 \\
\hline 5 & 84.5 & 117 & 83 & 114.7 & 6.4 & -14.7 & 6.5 & -15.9 \\
\hline 6 & 98.6 & 128.2 & 92.5 & 118.4 & 6.0 & -9.6 & 6.1 & -12.5 \\
\hline 7 & 80.8 & 150.1 & 81.3 & 106.7 & 6.1 & -12.0 & 6.4 & -15.7 \\
\hline 8 & 105.1 & 159.9 & 78.7 & 103 & 6.8 & -11.4 & 7.1 & -13.5 \\
\hline
\end{tabular}


Table III

\begin{tabular}{|c|c|c|c|c|c|}
\hline Ref. & $\begin{array}{c}\text { Band- } \\
\text { width }\end{array}$ & $\begin{array}{c}\text { Return } \\
\text { Loss }(\mathrm{dB})\end{array}$ & $\begin{array}{c}\text { Gain } \\
(\mathrm{dBi})\end{array}$ & Length & Polarization \\
\hline$[12]$ & $52 \%$ & $>14$ & $>7$ & $0.64 \lambda_{\mathrm{o}}$ & Dual \\
\hline$[13]$ & $75.3 \%$ & $>10$ & $>5$ & $0.53 \lambda_{\mathrm{o}}$ & Dual \\
\hline$[14]$ & $45 \%$ & $>15$ & $>6$ & $0.81 \lambda_{\mathrm{o}}$ & Dual \\
\hline$[15]$ & $41 \%$ & $>13$ & $>5.5$ & $1.2 \lambda_{\mathrm{o}}$ & Circular \\
\hline$[16]$ & $31.7 \%$ & $>10$ & $>7$ & $0.8 \lambda_{\mathrm{o}}$ & Circular \\
\hline $\begin{array}{c}\text { Tightly-fed } \\
\text { bowtie }\end{array}$ & $66.7 \%$ & $>15$ & $>6$ & $0.5 \lambda_{\mathrm{o}}$ & Dual \\
\hline $\begin{array}{c}\text { Capped } \\
\text { bowtie }\end{array}$ & $66.7 \%$ & $>15$ & $>6.1$ & $0.5 \lambda_{\mathrm{o}}$ & Dual \\
\hline
\end{tabular}

$\lambda_{\mathrm{o}}$ is the wavelength at the center frequency

\section{CONCLUSION}

In this paper, general bowtie antenna element candidates for phased arrays that can be applied in both communication system and radio astronomy are proposed. A tightly fed bowtie is designed with reflection coefficient better than -15 $\mathrm{dB}$ over an octave bandwidth. In order to improve the radiation properties, an improved capacitive loading bowtie dipole is developed. Prototype of the tightly fed and capacitive loading bowtie element are built and validate the superiority of the proposed antennas. Both of the simulation and measurement show the proposed UWB bowtie antennas have good properties for phased array application.

\section{REFERENCES}

[1] P. E. Dewdney, P. J. Hall, R. T. Schilizzi, and T. J. W. Lazio, "The Square Kilometre Array," Proc. IEEE, vol. 97, pp. 1482-1496, 2009

[2] Z. C. Zhang, H. W. Liu, S. W. Wong, "Compact wideband bandpass filter based on double-T-shaped stub loaded resonator and loading technique for zero-voltage point". Int J RF Microw Comput Aided Eng. 2018;28:e21197. https://doi.org/10.1002/ mmce.21197

[3] B. Veidt, G. Hovey, T. Burgess, R. Smegal, et al., "Demonstration of a dual-polarized phased-array feed," IEEE Trans. Antennas Propag., vol. 59, pp. 2047-2057, 2011.

[4] J. Fan et al., "Design of Octave-bandwidth Phased Array Feed for Large Radio Telescope," 2019 13th European Conference on Antennas and Propagation (EuCAP), Krakow, Poland, 2019, pp. 1-4.

[5] M. C. van Beurden et al., "Analysis of wideband infinite phased arrays of printed folded dipoles embedded in metallic boxes," IEEE Trans. Antennas Propag., vol. 48, no. 5, pp. 784-789, May 2000.

[6] W. S. T. Rowe, R. B. Waterhouse, and C. T. Huat, "Performance of a scannable linear array of Hi-Lo stacked patches," IEE Proc. Microwave, Antennas Propag., vol. 150, no. 1, pp. 1-4, Feb. 2003

[7] C. Wang, H. Liu, X. Zhang, S. Zhu, et al., "Single-feed wideband circularly polarized patch antenna using dual mode defected ground waveguide coupling structure." Int J RF Microw Comput Aided Eng. 2019;29:e21494. https://doi.org/10.1002/mmce.21494

[8] M. V. Ivashina, M. N. M. Kehn, P. S. Kildal, et al., "Decoupling efficiency of a wideband Vivaldi focal plane array feeding a reflector antenna," IEEE Trans. Antennas Propag., vol. 57, no. 2, pp. 373-382, Feb. 2009.

[9] R. W. Kindt and W. R. Pickles, "Ultrawideband all-metal flared notch array radiator," IEEE Trans. Antennas Propag., vol. 58, no. 11, pp. 3568-3575, Nov. 2010.

[10] H. Holter, "Dual-polarized broadband array antenna with BORelements, mechanical design and measurements," IEEE Trans. Antennas Propag., vol. 55, no. 2, Feb. 2007.

[11] W. F. Moulder, K. Sertel, and J. L. Volakis, "Superstrate-enhanced ultrawideband tightly coupled array with resistive FSS, " IEEE Trans. Antennas Propag., vol. 60, no. 9, pp. 4166 - 4172, 2012.

[12] Y. Gou, S. Yang, J. Li, and Z. Nie, “A compact dual-polarized printed dipole antenna with high isolation for wideband base station applications," IEEE Trans. Antennas Propag., vol. 62, no. 8, pp. 43924395, Aug. 2014.

[13] J. A. Kasemodel and J. L. Volakis, "A Planar Dual Linear-Polarized Antenna With Integrated Balun," IEEE Antennas and Wireless Propagation Letters, vol. 9, pp. 787-790, 2010.

[14] Y. Cui, R. Li and H. Fu, "A Broadband Dual-Polarized Planar Antenna for $2 \mathrm{G} / 3 \mathrm{G} / \mathrm{LTE}$ Base Stations," in IEEE Transactions on
Antennas and Propagation, vol. 62, no. 9, pp. 4836-4840, Sept. 2014, doi: 10.1109/TAP.2014.2330596.

[15] K. M. Mak and K. M. Luk, "A Circularly Polarized Antenna With Wide Axial Ratio Beamwidth," in IEEE Transactions on Antennas and Propagation, vol. 57, no. 10, pp. 3309-3312, Oct. 2009, doi: 10.1109/TAP.2009.2029370.

[16] Z. Li, J. Liu and Y. Long, "A Quasi-Magnetic-Electric Circularly Polarized Antenna With Wide Bandwidth," in IEEE Antennas and Wireless Propagation Letters, vol. 18, no. 10, pp. 2145-2149, Oct. 2019, doi: 10.1109/LAWP.2019.2938873. 